\title{
Local Synchronization of Sampled-Data Systems on One-Parameter Lie Subgroups
}

\author{
Philip James McCarthy
}

\begin{abstract}
We present a distributed nonlinear control law for synchronization of identical agents on one-parameter Lie subgroups. If the agents are initialized sufficiently close to one another, then synchronization is achieved exponentially fast. The proof does not use Jacobian linearization, instead the local nature of our result stems from our use of exponential coordinates on a matrix Lie group. We characterize all equilibria of the network and provide a characterization of deadbeat performance for a complete connectivity graph.
\end{abstract}

\section{INTRODUCTION}

Systems on matrix Lie groups are common in engineering. Rotational dynamics, such as those of UAVs, evolve on $\mathrm{SO}(3)$ or $\mathrm{SO}(2)$; if translational dynamics are also considered, they evolve on $\mathrm{SE}(3)$ [1] or $\mathrm{SE}(2)$ [2], respectively. Mobile ground robots can be modeled on $\mathrm{SE}(2)$, and quantum systems on $\mathrm{SU}(n)$ [3], [4]. The celebrated Kuramoto model of oscillator network evolves on the circle [5], which is isomorphic to $\mathrm{SO}(2)$.

The study of control theory on Lie groups began as early as the 1970s. The controllability of such systems was addressed in [6] and [7]; the latter also addressed observability and realization theory. We refer the reader to [8] for a more recent comprehensive treatment of control theory on Lie groups. Control theory on Lie groups differs from classical control because the state space is not a vector space. In the nonlinear context, such systems are usually controlled using differential geometric techniques, i.e., using coordinate charts on the Lie group to represent the system dynamics in local coordinates as systems in a vector space. Consequently, singularities arise from the choice of local coordinates as opposed to being intrinsic to the system's dynamics.

Control on Lie groups has also been treated globally, for example, motion tracking in $\mathrm{SE}(3)$ [9], the control of UAV [10] and spacecraft [11] orientation on $\mathrm{SO}(3)$, and the synchronization of networks of rigid bodies on $\mathrm{SE}(3)$ [12].

A framework for coordinated motion on Lie groups was developed in [13], where the synchronization problem that we consider is a special case of what the authors call biinvariant coordination. However, synchronization has been studied mostly for specific Lie groups, such as SE(3) [12]. The Kuramoto model was extended to $\mathrm{U}(n)$ in [14].

The sampled-data setup, i.e., a continuous-time plant and a discrete-time controller, is ubiquitous in applied control systems. In practice, controllers are often designed in

This research is supported by the Natural Sciences and Engineering Research Council of Canada (NSERC).

The authors are with the Dept. of Electrical and Computer Engineering, University of Waterloo, Waterloo ON, N2L 3G1 Canada. \{philip. mccarthy; cnielsen\}@uwaterloo.ca

\author{
Christopher Nielsen
}

continuous-time and it is assumed that the sampling period is small enough that the sampled-data behaviour will adequately match the theoretical continuous-time behaviour. But, in general, such setups are not guaranteed to even be stable for arbitrary sampling periods [15].

In the LTI case, the trajectory of the sampled-data system has an easily computed analytic solution. But closedform solutions to the sampled discrete-time dynamics of nonlinear systems do not exist, in general. Right invariant systems on matrix Lie groups are an exception, in that their sampled discrete-time time dynamics have closed-form solutions [16]. The closely related class of bilinear systems has received some attention in the discrete-time [16] and sampled-data settings [17]. In [18], passivity was used to achieve synchronized path-following for a class of nonlinear systems for small sampling periods. Passivity was also used to achieve synchronization under sampling for a network of Kuramoto-like oscillators in [19]. Synchronization of networks of harmonic oscillators under sampling with a timevarying period was investigated in [20].

We present a discrete-time control law that achieves synchronization for a sampled-data network of identical agents on one-parameter Lie subgroups with driftless dynamics. Networks of oscillators, which evolve on $\mathrm{SO}(2)$, are an example of such systems. If the agents are initialized sufficiently close to one another, then synchronization is achieved exponentially fast. We consider one-parameter subgroups to simplify analysis and facilitate discussion. We extend our results in the currently under-review paper [21]. These extensions include synchronization on any $m$-dimensional matrix Lie group and weighted connectivity graphs.

\section{A. Notation and Terminology}

Given $N \in \mathbb{N}$, let $\mathbb{N}_{N}:=\{1, \ldots, N\}$. Given a matrix $M \in \mathbb{C}^{n \times n}, M^{\top}$ is its (non-Hermitian) transpose. The Euclidean norm of $x \in \mathbb{C}^{n}$ is written $\|x\|$; similarly, the induced Euclidean norm of $M \in \mathbb{C}^{n \times n}$ is denoted by $\|M\|$. Let $\mathbf{1}_{n} \in \mathbb{R}^{n}$ and $\mathbf{0}_{n} \in \mathbb{R}^{n}$ denote the column vector of ones and zeros, respectively. Let $\mathbf{0}_{m \times n} \in \mathbb{R}^{m \times n}$ denote the matrix of zeros. Given $z \in \mathbb{C}$, let $z^{*}$ denote its complex conjugate. Let $\mathbb{R}^{-}$denote the set of nonpositive real numbers.

Unweighted, directed graphs are used to model communication constraints between agents. A graph $\mathcal{G}$ is a pair $(\mathcal{V}, \mathcal{E})$ consisting of a finite set of vertices $\mathcal{V}=\mathbb{N}_{N}$ and a set of edges $\mathcal{E} \subseteq \mathcal{V} \times \mathcal{V}$. Vertex $i$ 's neighbour set $\mathcal{N}_{i}:=\left\{j \in \mathbb{N}_{N}\right.$ : $(i, j) \in \mathcal{E}\}$. If agent $i$ has access to its relative state with respect to agent $j$, then $(i, j) \in \mathcal{E}$. We assume that $\mathcal{G}$ has no self-loops. 


\section{SAMPled-DAta SynChronization Problem}

We consider a network of $N$ controlled agents, each modelled by the differential equation

$$
\dot{X}_{i}=X_{i}\left(\sum_{j=1}^{m} B_{i, j} u_{i, j}\right), \quad i \in \mathbb{N}_{N} .
$$

Here $X_{i} \in \mathrm{G}$ where $\mathrm{G} \subset \mathrm{GL}(n, \mathbb{C})$ is an $m$-dimensional connected matrix Lie group over the complex field $\mathbb{C}$ which includes, as a special case, real matrix Lie groups. The matrices $B_{i, j}$ are elements of the Lie algebra $\mathfrak{g}$, which is a vector space over a field $\mathbb{F}$ equal to either $\mathbb{C}$ or $\mathbb{R}$, associated with $\mathrm{G}$, and $u_{i}=\left(u_{i, 1}, \ldots, u_{i, m}\right) \in \mathbb{F}^{m}$ is the control input. Note that the Lie algebra of a complex Lie group $G$ may in fact be a real vector space. For example, the Lie algebra $\mathfrak{s u}(2)$ of the complex Lie group $\mathrm{SU}(2)$ is a vector space over the field of reals despite its vectors being matrices with possibly non-real entries. Equation (1) is a kinematic model of a system evolving on a matrix Lie group G. Each agent is assumed to be fully actuated in the sense that

$$
\left(\forall i \in \mathbb{N}_{N}\right) \operatorname{span}_{\mathbb{F}}\left\{B_{i, 1}, \ldots, B_{i, m}\right\}=\mathfrak{g} .
$$

Under this assumption, without loss of generality, we take the system (1) to be driftless since the inputs $u_{i}, i \in \mathbb{N}_{N}$, can be chosen to cancel any drift vector field. We are interested in the sampled-data control of this multi-agent system in which each agent's control law is implemented on an embedded computer, which we explicitly model using the setup in Figure 1. The blocks $H$ and $S$ in Figure 1 are, respectively, the ideal hold and sample operators. Sample and hold represent $\mathrm{A} / \mathrm{D}$ and $\mathrm{D} / \mathrm{A}$ conversion, respectively. The following assumption is made throughout this paper.

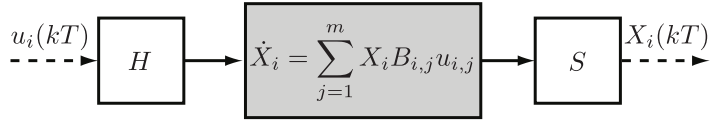

Fig. 1: Sampled-data agent on a matrix Lie group G.

Assumption 1. All sample and hold blocks operate at the same period $T>0$ and the blocks are synchronized for the multi-agent system (1).

Under Assumption 1, letting $X_{i}[k]:=X_{i}(k T)$ and $u_{i}[k]:=u_{i}(k T)$, the discretized dynamics of each agent are

$$
X_{i}^{+}=X_{i} \exp \left(T \sum_{j=1}^{m} B_{i, j} u_{i, j}\right), \quad i \in \mathbb{N}_{N}
$$

which is an exact discretization of (1). For each $i \in \mathbb{N}_{N}$, define $\Omega_{i}:=\sum_{j=1}^{m} B_{i, j} u_{i, j} \in \mathfrak{g}$. Then the discrete-time dynamics can be compactly expressed as

$$
X_{i}^{+}=X_{i} \exp \left(T \Omega_{i}\right), \quad i \in \mathbb{N}_{N}
$$

\section{A. The Synchronization Problem}

Given a network of $N$ agents with kinematic dynamics (3), we define the error quantities $E_{i j}:=X_{i}^{-1} X_{j}, i, j \in \mathbb{N}_{N}$. Observe that $E_{i j}=I$ if, and only if, $X_{i}=X_{j}$. The error matrix $E_{i j}$ is called right invariant [22] since, for all $X \in$ $\mathrm{G},\left(X X_{i}\right)^{-1}\left(X X_{j}\right)=X_{i}^{-1} X_{j}$. Since the class of systems considered has $\mathrm{G}$ for its state space, which is generally not a vector space, we do not use $X_{i}-X_{j}$ as a measure of error.

Local Synchronization on Matrix Lie Groups : Given a network of $N$ agents with continuous-time dynamics (1), sampling period $T>0$ and an unweighted, connected communication graph $\mathcal{G}=(\mathcal{V}, \mathcal{E})$, find, if possible, distributed control laws $\Omega_{i}, i \in \mathbb{N}_{N}$, such that for all initial conditions in a neighbourhood of the identity in $\mathrm{G}^{N}$, for all $i, j \in \mathbb{N}_{N}$, $E_{i j} \rightarrow I$ as $t \rightarrow \infty$.

By a distributed control law we mean that for each agent $i$, the control signal $\Omega_{i}$ depends on $E_{i j}$ only if $(i, j) \in \mathcal{E}$. We propose the distributed feedback control law

$$
\Omega_{i}:=\frac{1}{T} \log \left(\left(\prod_{j \in \mathcal{N}_{i}} E_{i j}\right)^{\frac{1}{K}}\right)
$$

where $K \in \mathbb{R}$ is a gain and the matrix logarithm need not be the principal logarithm. The control law (4) does not require agent $i$ to know agent $j$ 's state $X_{j}$, or even its own state $X_{i}$, but instead requires knowledge of the relative state $E_{i j}$. The expression (4) is well-defined so long as the product $\prod_{j \in \mathcal{N}_{i}} E_{i j}$ has no eigenvalues in $\mathbb{R}^{-}$, as discussed in Section III. This control law is defined in global coordinates, but we only prove local exponential stability of the synchronized state. When the control law (4) is welldefined, the closed-loop discrete-time dynamics are

$$
X_{i}^{+}=X_{i}\left(\prod_{j \in \mathcal{N}_{i}} E_{i j}\right)^{\frac{1}{K}}, \quad i \in \mathbb{N}_{N}
$$

and it follows from the definition of $E_{i j}$ that

$$
E_{i j}^{+}=\left(\prod_{p \in \mathcal{N}_{i}} E_{i p}\right)^{-\frac{1}{K}} E_{i j}\left(\prod_{q \in \mathcal{N}_{j}} E_{j q}\right)^{\frac{1}{K}} .
$$

Remark II.1. The order of multiplication in (4) need not be common to all agents or even constant.

This control law (4) is motivated by exponential coordinates for Lie groups, classical consensus algorithms in $\mathbb{R}^{n}$, and the notion of Riemannian mean of rotations on $\mathrm{SO}(3)$, which on a one-parameter subgroup thereof can be explicitly computed as $\prod_{i=1}^{N} R_{i}^{1 / N}$ [23].

A key advantage of direct design over emulation, is that stability can be guaranteed at the sampling instants. This does not necessarily imply good performance between sampling instants, however. But in the specific case of the plant and problem discussed in this paper, achieving synchronization at the sampling instants implies synchronization between the sampling instants. 
Proposition II.2. If $E_{i j}[k]=X_{i}[k]^{-1} X_{j}[k]$ asymptotically approaches $I$ as $k \rightarrow \infty$, then $E_{i j}(t)=X_{i}(t)^{-1} X_{j}(t)$ asymptotically approaches $I$ as $t \rightarrow \infty$, where $X_{i}(t)$ and $X_{j}(t)$ evolve according to (1).

Proof. If $E_{i j}[k] \rightarrow I$, then the proposed control law (4) satisfies $\Omega_{i}[k] \rightarrow \mathbf{0}_{n \times n}$. Let $0<\delta<T$. Then

$$
\begin{aligned}
\lim _{k \rightarrow \infty} & E_{i j}(k T+\delta)=\lim _{k \rightarrow \infty} \exp \left(\delta \Omega_{i}[k]\right)^{-1} E_{i j}[k] \exp \left(\delta \Omega_{j}[k]\right) \\
& =\lim _{k \rightarrow \infty} \exp \left(\delta \Omega_{i}[k]\right)^{-1} \lim _{k \rightarrow \infty} E_{i j}[k] \lim _{k \rightarrow \infty} \exp \left(\delta \Omega_{j}[k]\right) \\
& =I^{3}=I .
\end{aligned}
$$

Since $\delta$ is arbitrary, this implies that $E_{i j}(t) \rightarrow I$.

Proposition II.2 means that asymptotically stabilizing the set where $E_{i j}=I$, for all $i, j \in \mathbb{N}_{N}$, at the sampling instants is sufficient to solve the synchronization problem. Thus, we can conduct all analysis in the discrete-time setting and do not rely on $T$ being sufficiently small. We impose the following assumption, which greatly simplifies analysis.

Assumption 2. For all $i, j \in \mathbb{N}_{N}$, we have $E_{i j} \in \mathrm{H}$, for some one-parameter subgroup $\mathrm{H}$ of $\mathrm{G}$.

\section{PRELIMINARIES}

\section{A. Functions of matrices}

For every nonsingular matrix $X \in \mathbb{C}^{n \times n}$ there are (infinitely many) $A \in \mathbb{C}^{n \times n}$ such that $\exp (A)=X$, see [24, Theorem 1.27]. Every such matrix $A$ is a nonprimary logarithm of $X$, which we denote by $\log (X)$. If, in addition to being nonsingular, the matrix $X$ has no eigenvalues in $\mathbb{R}^{-}$, then it has a principal logarithm.

Theorem III.1 ([24, Theorem 1.31]). Let $X \in \mathbb{C}^{n \times n}$ have no eigenvalues in $\mathbb{R}^{-}$. There is a unique logarithm $A \in$ $\mathbb{C}^{n \times n}$ of $X$, all of whose eigenvalues lie in the strip $\{z$ : $-\pi<\operatorname{Im}(z)<\pi\}$. If $X \in \mathbb{R}^{n \times n}$, then $A \in \mathbb{R}^{n \times n}$.

The unique matrix $A$ from Theorem III.1 is called the principal logarithm of $X$ and is denoted by $\log (X)$. Unlike complex numbers, it is not possible to express $\log (X)$ as a function of $\log (X)$ for arbitrary non-singular matrices. If $\|X-I\|<1$ then

$$
\log (X)=\sum_{k=1}^{\infty} \frac{(-1)^{k-1}}{k}(X-I)^{k} .
$$

Borrowing from the definitions of complex powers of scalars [25, Chapter III, $\S 6]$ and the form of the square root of a matrix on a Lie group [26, Lemma 2.14], we define the $K$ th root of a matrix in the following way.

Definition III.2. Let $X \in \mathbb{C}^{n \times n}$ have no eigenvalues in $\mathbb{R}^{-}$. Given $K \in \mathbb{R}$, the principal Kth root of $X$ is

$$
X^{\frac{1}{K}}:=\exp \left(\frac{1}{K} \log (X)\right) .
$$

If $X \in \mathrm{G}$, then $X^{1 / K} \in \mathrm{G}$, due to the Lie correspondence $\log : \mathrm{G} \rightarrow \mathfrak{g}$. We allow only $K \geq 1$.
Remark III.3. If $X^{1 / K}$ is well-defined, then for $K \in \mathbb{N}$,

$$
\left(X^{\frac{1}{K}}\right)^{K}=\exp \left(\sum_{i=1}^{K} \frac{1}{K} \log (X)\right)=\exp (\log (X))=X .
$$

Thus, in this case, $X=X^{1 / K} X^{1 / K} \cdots X^{1 / K}$ ( $K$ times $)$, which is the intuitive notion of a Kth root. The somewhat indirect definition (8) allows for Kth roots for $K \in \mathbb{R}$.

Throughout this paper, we use an important algebraic property of the logarithm of a matrix power.

Theorem III.4 ([24, Theorem 11.2]). If $X \in \mathbb{C}^{n \times n}$ has no eigenvalues in $\mathbb{R}^{-}$, then for $\alpha \in[-1,1]$, we have $\log \left(X^{\alpha}\right)=\alpha \log (X)$.

\section{B. Exponential Coordinates and One-Parameter Subgroups}

The exponential map exp $: \mathfrak{g} \rightarrow \mathrm{G}$ can be used to define coordinates on $\mathrm{G}$. Choose $r>0$ so that (7) converges on $\{X \in \mathrm{G}: X=\exp (A),\|A\|<r\}$, e.g., $r=\log (2)$. Larger values of $r$ may be possible for specific groups. The set

$$
U:=\{X \in \mathrm{G}: X=\exp (A), A \in \mathfrak{g},\|A\|<r\}
$$

is an open neighbourhood of $I$ in $\mathrm{G}$ in the group topology in which $\log : U \rightarrow \mathfrak{g}$ provides an inverse. Fix a basis $\left\{A_{1}, A_{2}, \ldots, A_{m}\right\}$ for $\mathfrak{g}$. Define the isomorphism

$$
\vee: \mathfrak{g} \rightarrow \mathbb{F}^{m}, \quad H \mapsto\left[\begin{array}{llll}
t_{1} & t_{2} & \cdots & t_{m}
\end{array}\right]^{\top},
$$

where $t \in \mathbb{F}^{m}$ is the unique vector for which $H=t_{1} A_{1}+$ $t_{2} A_{2}+\cdots+t_{m} A_{m}$. We have the mapping diagram

$$
U \subset \mathrm{G} \stackrel{\log }{\longrightarrow} B_{r}(0) \subseteq \mathfrak{g} \stackrel{\vee}{\longrightarrow} V \subseteq \mathbb{F}^{m}
$$

where $B_{r}(0):=\{A \in g:\|A\|<r\}$ is the image of $U$ under Log. Denote by $\psi: U \rightarrow V$ the composition $\vee \circ$ Log. The coordinates $\left(t_{1}, \ldots, t_{m}\right) \in \mathbb{F}^{m}$ are the exponential coordinates of the first kind on $\mathrm{G}$.

Thus, a neighbourhood of the identity in a Lie group $G$ can be identified with an open subset of the vector space $\mathbb{F}^{m}$ containing the origin. We hereinafter refer to these coordinates as simply "exponential coordinates".

Definition III.5 (One-Parameter Subgroup). Given a Lie group $\mathrm{G}$, a one-parameter subgroup is a continuous morphism of groups $\phi: \mathbb{R} \rightarrow \mathrm{G}$.

Although this terminology is standard, it is technically the image of the map $\phi$ that is a subgroup of G. The subgroup $\mathrm{H}:=\phi(\mathbb{R}) \subset \mathrm{G}$ is a one-dimensional manifold and there exists a unique $H \in \mathfrak{g}$ such that $\phi(\theta)=\exp (\theta H)$ for all $\theta \in \mathbb{R}[26$, Theorem 2.13].

In the case of a one-parameter subgroup $\mathrm{H}$, exponential coordinates admit a local identification of $\mathrm{H} \cap U$ with an open interval of $\mathbb{R}$ containing the origin. Fix a basis $\left\{A_{1}, \ldots, A_{m}\right\}$ of $\mathfrak{g}$. If $H \in \mathfrak{g}$, then there exists a unique $t \in \mathbb{F}^{m}$ such that $H=\sum t_{i} A_{i}$. Therefore, the exponential coordinate map is simplified and is given by $\psi(X)=\theta t=\theta\left[\begin{array}{lll}t_{1} & \cdots & t_{m}\end{array}\right]^{\top}$. That is, if $X \in \mathrm{H} \cap U$, then $\psi(X)$ is an element of the onedimensional subspace $\operatorname{span}_{\mathbb{R}}\{t\} \subset \mathbb{F}^{m}$. We can therefore locally identify $X \in \mathrm{H} \cap U$ with the parameter $\theta \in \mathbb{R}$. 


\section{SYNCHRONIZATION}

Let $\phi: \mathbb{R} \rightarrow \mathrm{G}$ be a one-parameter subgroup, $\mathrm{H}=\phi(\mathbb{R})$. By Assumption 2, for all $i, j \in \mathbb{N}_{N}, E_{i j} \in \mathrm{H}$. If $X_{i}, X_{j} \in \mathrm{H}$, then $E_{i j}=X_{i}^{-1} X_{j} \in \mathrm{H}$. Let $\theta_{i j}:=\phi^{-1}\left(E_{i j}\right)$.

Proposition IV.1. Any one-parameter subgroup $\mathrm{H} \subset \mathrm{G}$, on which (4) is well-defined, is positively invariant for (6).

Proof. Let $\mathrm{H} \subset \mathrm{G}$ be a one-parameter subgroup generated by $H \in \mathfrak{g}$. Let $k \in \mathbb{Z}$ be arbitrary and suppose that for all $i, j \in$ $\mathbb{N}_{N}, E_{i j}[k] \in \mathrm{H}$. Let $q \in \mathbb{N}_{N}$ be arbitrary. By elementary group theory $\prod_{j \in \mathcal{N}_{q}} E_{q j}[k] \in \mathrm{H}$ and therefore, by hypothesis, its $K$ th root is well-defined. Thus, by definition of log, $\exp \left(T \Omega_{q}[k]\right) \in \mathrm{H}$. Since $E_{i j}^{+}=\exp \left(-T \Omega_{i}\right) E_{i j} \exp \left(T \Omega_{j}\right)$, we have $E_{i j}[k+1] \in \mathrm{H}$. Induction on the time index proves positive invariance of $\mathrm{H}$.

Note that $X_{i} \in \mathrm{H}$ for all $i \in \mathbb{N}_{N}$ is merely a sufficient condition for $E_{i j} \in \mathrm{H}$, for all $i, j \in \mathbb{N}_{N}$. Using the exponential coordinates from Section III-B we identify each relative error $E_{i j} \in \mathrm{H} \cap U$ with their scalar coordinates $\theta_{i j} \in \mathbb{R}$. The map $\phi$ is a diffeomorphism in a neighbourhood of the identity, yielding

$$
\theta_{i j}^{+}=\left(-\frac{1}{K} \sum_{p \in \mathcal{N}_{i}} \theta_{i p}\right)+\theta_{i j}+\left(\frac{1}{K} \sum_{q \in \mathcal{N}_{j}} \theta_{j q}\right) .
$$

For all $i, j \in \mathbb{N}_{N}, E_{i j}=E_{1 i}^{-1} E_{1 j}$, so (9) can be rewritten as

$$
\begin{aligned}
\theta_{i j}^{+} & =\theta_{i j}-\frac{1}{K} \sum_{p \in \mathcal{N}_{i}}\left(\theta_{1 p}-\theta_{1 i}\right)+\frac{1}{K} \sum_{q \in \mathcal{N}_{j}}\left(\theta_{1 q}-\theta_{1 j}\right) \\
& =\theta_{i j}+\frac{1}{K}\left(\ell_{i}-\ell_{j}\right) \Theta,
\end{aligned}
$$

where $\ell_{i}$ and $\ell_{j}$ are rows $i$ and $j$, respectively, of the Laplacian $L$ of the communication graph $\mathcal{G}$. Setting $i=1$ and "stacking" the last line for all $j$, we obtain

$$
\Theta^{+}=\left(I+\frac{1}{K}\left(\mathbf{1}_{N} \ell_{1}-L\right)\right) \Theta,
$$

where $\Theta:=\left[\begin{array}{llll}\theta_{11} & \theta_{12} & \cdots & \theta_{1 N}\end{array}\right]^{\top}$. Thus, the local error dynamics are linear. The linear dynamics (10) are (exponentially) stable if and only if the matrix $I+\left(\mathbf{1}_{N} \ell_{1}-L\right) / K$ is Schur. We must therefore establish conditions on the gain $K$ such that all eigenvalues of $I+\left(\mathbf{1}_{N} \ell_{1}-L\right) / K$ are in the open unit disc.

The Laplacian $L$ of the graph $\mathcal{G}$ is positive semidefinite, with a zero eigenvalue of algebraic multiplicity equal to the number of connected components in $\mathcal{G}$ [27, Lemma 13.1.1]; the eigenvector associated with the 0 eigenvalue is $\mathbf{1}_{N}$.

Lemma IV.2. The spectrum of $\mathbf{1}_{N} \ell_{1}-L$ equals $\sigma(-L)$.

Proof. Let $J$ be the Jordan form of $L$ and let $V \in \mathbb{C}^{N \times N}$ be the nonsingular matrix such that $J=V^{-1} L V$, where the first column $V_{1}$ is in the span of $\mathbf{1}_{N}$. We have

$$
V^{-1}\left(\mathbf{1}_{N} \ell_{1}-L\right) V=V^{-1} \mathbf{1}_{N} \ell_{1} V-J .
$$

Since $V_{1}$ is in the span of $\mathbf{1}_{N}$ and $V^{-1} V=I$, we have $\left(V^{-1} \mathbf{1}_{N}\right)_{i}=0$ for all $i \neq 1$. Also because $V_{1}$ is in the span of $\mathbf{1}_{N}$, we have $\ell_{1} V_{1}=0$, so $\left(V^{-1} \mathbf{1}_{N}\right)_{i}=0$ for all $i \neq 1$. Therefore, $V^{-1} \mathbf{1}_{N} \ell_{1} V$ is strictly upper triangular. Therefore, the eigenvalues of (11) are its diagonal elements, which are the diagonal elements of $-J$, which are the negatives of the eigenvalues of $L$.

Lemma IV.3. The spectrum of $I+\left(\mathbf{1}_{N} \ell_{1}-L\right) / K$ is the image of $1-\sigma(L) / K$.

Proof. The result follows from Lemma IV.2 and applying the Spectral Mapping Theorem [24, Theorem 1.13] using the function $f: \mathbb{C} \rightarrow \mathbb{C}, f(x)=1-x / K$.

Since the graph is assumed to be connected, $L$ has a simple eigenvalue at 0 . By Lemma IV.3, this eigenvalue gets mapped to 1 in the spectrum of $I+\left(\mathbf{1}_{N} \ell_{1}-L\right) / K$.

Let $\lambda$ be an eigenvalue of $L$ and define the function $f(x)=$ $1-x / K$ as in the proof of Lemma IV.3, then

$f(\lambda)=1-\frac{|\lambda|}{K} \mathrm{e}^{j \measuredangle \lambda}=\left(1-\frac{|\lambda|}{K} \cos (\measuredangle \lambda)\right)-j \frac{|\lambda|}{K} \sin (\measuredangle \lambda)$

For stability, we require $f(\lambda)$ to be in the open unit disc. Since we have already addressed the single eigenvalue at 0 , we assume that $\lambda \neq 0$. Straightforward arithmetic and the quadratic formula verify that all all eigenvalues of $I+$ $\left(\mathbf{1}_{N} \ell_{1}-L\right) / K$, except the simple eigenvalue at 1 , are in the open unit disc if $K$ satisfies

$$
(\forall \lambda \in \sigma(L) \backslash\{0\}) \quad K>\frac{|\lambda|}{2 \cos (\measuredangle \lambda)}=\frac{|\lambda|^{2}}{2 \operatorname{Re}(\lambda)} .
$$

It is possible to lower bound $K$ as a function of the number of agents $N$, using the properties of the eigenvalues of the Laplacian of a directed graph [28].

Lemma IV.4. If $K>K_{\min }(N)$, where

$$
K_{\min }(N):=\left\{\begin{aligned}
& \frac{N}{2} N \leq 9, \\
& \frac{1}{8} \csc ^{2}\left(\frac{\pi}{2 N}\right) \sec \left(\frac{\pi}{N}\right) 10 \leq N \leq 18, \\
& N-1 \quad N \geq 19,
\end{aligned}\right.
$$

then $I+\left(\mathbf{1}_{N} \ell_{1}-L\right) / K$ has a simple eigenvalue at 1 and all others in the open unit disc.

Proof. Due to lack of space, we refer the reader to [21].

Remark IV.5. If $\mathcal{G}$ is symmetric, then $\sigma(L) \subset[0, N]$, thus (12) in Lemma IV.4 simplifies to $K_{\min }(N)=N / 2$.

By Lemma IV.4, there is no $K$ for which $I+\left(\mathbf{1}_{N} \ell_{1}-L\right) / K$ is Schur. However, this does not preclude stability of (10), because the eigenvalue of 1 corresponds to the dynamics of $\theta_{11}$, the error of agent 1 with itself, which is identically 0 .

Theorem IV.6. If the gain $K$ of each agent's controller (4) satisfies (12), then the equilibrium $\Theta=\mathbf{0}_{N}$ of (10) is locally exponentially stable.

Proof. Since $E_{11}(t)=X_{1}^{-1}(t) X_{1}(t) \equiv I$, it immediately follows that $\theta_{11}(t) \equiv 0$. Therefore the $N-1$ dimensional subspace $\mathcal{V}:=\left\{\Theta \in \mathbb{R}^{N}: \theta_{11}=0\right\}$ is invariant under the dynamics (10). As a result, we have

$$
\sigma\left(I+\left(\mathbf{1}_{N} \ell_{1}-L\right) / K\right)=\sigma\left(I+\left(\mathbf{1}_{N} \ell_{1}-L\right) / K \mid \mathcal{V}\right) \sqcup\{1\}
$$


where $I+\left(\mathbf{1}_{N} \ell_{1}-L\right) / K \mid \mathcal{V}$ is the restriction of the system matrix in (10) to the subspace $\mathcal{V}$. If the gain $K$ of each agent's controller (4) satisfies (12), then by Lemma IV.4 $I+$ $\left(\mathbf{1}_{N} \ell_{1}-L\right) / K \mid \mathcal{V}$ is Schur.

Remark IV.7. Theorem IV.6 implies that there exists a positively invariant set in $\mathrm{H} \cap U$ containing the identity.

We emphasize that Theorem IV.6 does not rely on (Jacobian) linearization of the nonlinear dynamics $E_{i j}^{+}$. The system in exponential coordinates evolves according to linear dynamics. The locality of the result stems from the fact that the chart corresponding to exponential coordinates does not cover the entire manifold $\mathrm{G}$ nor the submanifold $\mathrm{H}$.

\section{EQUILIBRIA}

Since we consider kinematic models, the system is at equilibrium if and only if every agent's input is zero, i.e., for all $i \in \mathbb{N}_{N}, \Omega_{i}=\mathbf{0}_{n \times n}$. We provide a characterization of all equilibria and show that they are isolated. As in Section IV, we can perform our analysis in the one-parameter coordinates induced by the map $\phi: \mathbb{R} \rightarrow \mathrm{G}$. By definition, $\phi$ is surjective onto its image $\mathrm{H}$, but it is not necessarily injective. However, the restriction $\phi: \mathbb{R} / \operatorname{ker}(\phi) \rightarrow \mathrm{H}$ is bijective. Let $[\theta]$ denote the equivalence class defined by $\theta \sim \theta+k$, for all $k \in \operatorname{ker}(\phi)$.

Proposition V.1. If the controller (4) is well-defined, then the equilibria of (6) are $\left(\phi^{-1}\left(E_{11}\right), \ldots, \phi^{-1}\left(E_{1 N}\right)\right) \in\left[\mathbf{0}_{N}\right]$.

Proof. The sampled dynamics of each agent are given by (5). Therefore, the system is at equilibrium if and only if for all $i \in \mathbb{N}_{N}$, $\exp \left(T \Omega_{i}\right)=I$. On a one-parameter subgroup $\mathrm{H}$, by commutativity and Definition III.2, this condition becomes

$$
I=\exp \left(T \Omega_{i}\right)=\left(\prod_{j \in \mathcal{N}_{i}} E_{i j}\right)^{\frac{1}{K}}=\prod_{j \in \mathcal{N}_{i}} E_{1 i}^{-\frac{1}{K}} E_{1 j}^{\frac{1}{K}} .
$$

In one-parameter coordinates, we have

$$
\begin{aligned}
\phi^{-1}(I) & =\sum_{j \in \mathcal{N}_{i}}\left(\phi^{-1}\left(E_{1 i}^{-\frac{1}{K}}\right)+\phi^{-1}\left(E_{1 j}^{\frac{1}{K}}\right)\right) \\
{[0] } & =\frac{1}{K} \sum_{j \in \mathcal{N}_{i}}\left(\left[\theta_{1 j}\right]-\left[\theta_{1 i}\right]\right)=\frac{1}{K} \ell_{i}[\Theta],
\end{aligned}
$$

where $\Theta:=\left(\phi^{-1}\left(E_{11}\right), \ldots, \phi^{-1}\left(E_{1 N}\right)\right)$.

As in Section IV, we "stack" the inputs for all $i$, yielding

$$
L[\Theta]=\left[\mathbf{0}_{N}\right] .
$$

Since $\mathcal{G}$ is connected, $L \in \mathbb{Z}^{N \times N}$ has rank $N-1$ and its kernel is spanned by $\mathbf{1}_{N}$. Since (13) is homogeneous, $[\Theta]=$ $[0]$ is a solution. Thus all solutions to (13) are given by $[\Theta]=a\left[\mathbf{1}_{N}\right], a \in \mathbb{R}$. But, $\left[\theta_{11}\right]=[0]$, so $a=0(\bmod \operatorname{ker}(\phi))$. Therefore, the equilibria are given by $\Theta \in\left[\mathbf{0}_{N}\right]$.

Proposition V.2. All equilibria are isolated.

Proof. If $\operatorname{ker}(\phi)=\{0\}$, then $\mathbb{R} \backslash \operatorname{ker}(\phi) \cong \mathbb{R}$. Thus, $[\Theta]=$ $\left[\mathbf{0}_{N}\right]$ simplifies to $\Theta=\mathbf{0}_{N}$.

If $\operatorname{ker}(\phi) \neq\{0\}$, then let $d>0$ be the generator of $\operatorname{ker}(\phi)$. Then for every $r \in[0]$, there exists a $q \in \mathbb{Z}$, such that $r=q d$. Therefore, if $\Theta, \Theta^{\prime} \in\left[\mathbf{0}_{N}\right], \Theta \neq \Theta^{\prime}$, then $\left\|\Theta-\Theta^{\prime}\right\| \geq d$.

\section{Performance With a COMPlete Graph}

Proposition VI.1. If $\mathcal{G}$ is complete, then the $\varepsilon$ settling time, where $\varepsilon \in(0,1)$, is

$$
T_{s}=\left\lceil\frac{\log \varepsilon}{\log \left(\frac{|K-N|}{K}\right)}\right\rceil .
$$

Proof. Using the fact that on $U$, we have $\theta_{i j}+\theta_{j k}=\theta_{i k}$, if $\mathcal{G}$ is complete, then the local error dynamics (9) simplify to

$$
\theta_{i j}^{+}=\frac{K-N}{K} \theta_{i j}
$$

Therefore, the $\varepsilon$ settling time is computed thus

$$
\left|\frac{K-N}{K}\right|^{k}=\varepsilon \Longrightarrow k=\frac{\log \varepsilon}{\log \left(\frac{|K-N|}{K}\right)}
$$

Since $k$ is a time-step, we round up to the nearest integer.

The derivative of the settling time with respect to $K$ is

$$
\frac{\partial T_{s}}{\partial K}=\frac{-\log (\varepsilon) N}{K(K-N)\left(\log \left(\frac{|K-N|}{K}\right)\right)^{2}} .
$$

If $K>N$, then (15) is positive, so increasing $K$, i.e., reducing the gain $1 / K$, delays synchronization, which agrees with intuition. But, interestingly, if $K<N$, then (15) is negative, so increasing $K$ hastens synchronization.

Proposition VI.2. If $\mathcal{G}$ is complete and $K=N$, then synchronization is achieved at time-step $k=1$.

Proof. Setting $K=N$ in (14), we have $\theta_{i j}^{+}=0$.

\section{SIMULATION}

The Lie group $\mathrm{SO}(2)$ is one dimensional, thus, it is a one-parameter subgroup of $\mathrm{SO}(n)$ for any $n \geq 2$. $\mathrm{SO}(2)$ is the group of rotations in the plane, which can be interpreted locally as a position on the circumference of a circle. Given an element $R \in \mathrm{SO}(2)$, its local coordinate $\theta \in \mathbb{R}$ is often called the "phase" or "angle". The Kuramoto oscillator is a popular model of synchronization of networks of oscillators. We can view a Kuramoto network of $N$ agents as a control system, where agent $i$ has phase $\theta_{i} \in \mathbb{R}$ with dynamics

$$
\dot{\theta}_{i}=u_{i}, \quad u_{i}=-\sum_{j \in \mathcal{N}_{i}} a_{i j} \sin \left(\theta_{i}-\theta_{j}\right),
$$

where $a_{i j} \in \mathbb{R}$ is the coupling strength between agents $i$ and $j$. System (16) can be modelled as a system on a Lie group in the form of (1), where

$R_{i}=\phi(\theta)=\left[\begin{array}{cc}\cos (\theta) & -\sin (\theta) \\ \sin (\theta) & \cos (\theta)\end{array}\right], \quad \dot{R}_{i}=R_{i}\left[\begin{array}{cc}0 & -1 \\ 1 & 0\end{array}\right] u_{i}$

We simulate using $N=3$ and $a_{i j}=1$ for all $i, j \in \mathbb{N}_{N}$. It can be shown that with these parameters, that (16) achieves phase synchronization [5]. Sampling with period $T=0.8$, we see in Figure 2 that synchronization is destroyed. 


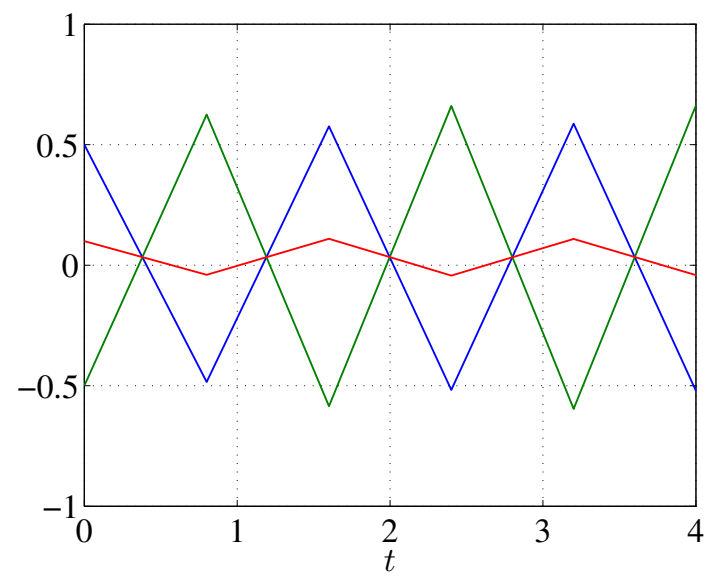

Fig. 2: Phases of sampled Kuramoto network with $T=0.8$.

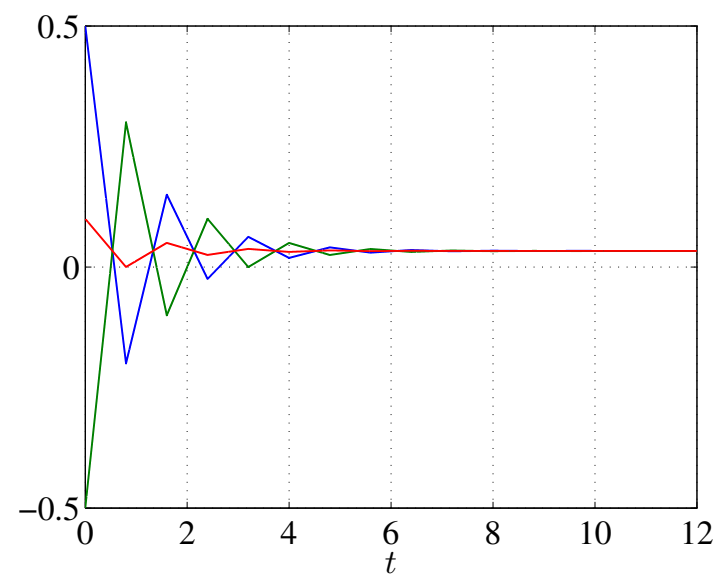

Fig. 3: Phases using proposed controller with $T=0.8$.

Guided by Theorem IV.6, we simulate this network again, with $T=0.8$, using the proposed controller (4) with $K=$ $2>N / 2=1.5$. Figure 3 illustrates that synchronization is achieved at $T=0.8$, whereas it was lost using the naïvely discretized Kuramoto coupling.

\section{FUTURE WORK}

Future work includes extending our results to agents with dynamic models and relaxing the assumption that the agents are fully actuated. The latter could first be addressed by assuming that the inputs generate the Lie algebra $\mathfrak{g}$.

\section{REFERENCES}

[1] A. Roza and M. Maggiore, "A Class of Position Controllers for Underactuated VTOL Vehicles," IEEE Transactions on Automatic Control, vol. 59, no. 9, pp. 2580-2585, 2014. 1

[2] E. Justh and P. Krishnaprasad, "Equilibria and steering laws for planar formations," Systems \& Control Letters, vol. 52, no. 1, pp. 25-38, 2004. 1

[3] C. Altafini and F. Ticozzi, "Modeling and Control of Quantum Systems: An Introduction," IEEE Transactions on Automatic Control, vol. 57, no. 8, pp. 1898-1917, 2012. 1

[4] F. Albertini and D. D'Alessandro, "Minimum time optimal synthesis for two level quantum systems," Journal of Mathematical Physics, vol. 56 , no. $1,2015.1$
[5] F. Dörfler and F. Bullo, "Synchronization in complex networks of phase oscillators: A survey," Automatica, vol. 50, no. 6, pp. 15391564, 2014. 1,5

[6] V. Jurdjevic and H. J. Sussmann, "Control systems on Lie groups," Journal of Differential Equations, vol. 12, no. 2, pp. 313-329, 1972. 1

[7] R. Brockett, "System Theory on Group Manifolds and Coset Spaces," SIAM Journal on Control, vol. 10, no. 2, pp. 265-284, 1972. 1

[8] Y. Sachkov, "Control theory on Lie groups," Journal of Mathematical Sciences, vol. 156, no. 3, pp. 381-439, 2009. 1

[9] J. Park and K. Kim, "Tracking on Lie group for robot manipulators," in International Conference on Ubiquitous Robots and Ambient Intelligence. Kuala Lumpur: IEEE, 2014, pp. 579-584. 1

[10] J. R. Forbes, "Passivity-Based Attitude Control on the Special Orthogonal Group of Rigid-Body Rotations," Journal of Guidance, Control, and Dynamics, vol. 36, no. 6, pp. 1596-1605, 2013. 1

[11] O. Egeland and J.-M. Godhavn, "Passivity-based adaptive attitude control of a rigid spacecraft," IEEE Transactions on Automatic Control, vol. 39, no. 4, pp. 842-846, 1994.1

[12] Y. Igarashi, T. Hatanaka, M. Fujita, and M. W. Spong, "Passivity-based attitude synchronization in SE(3)," IEEE Transactions on Control Systems Technology, vol. 17, no. 5, pp. 1119-1134, 2009. 1

[13] A. Sarlette, S. Bonnabel, and R. Sepulchre, "Coordinated Motion Design on Lie Groups," IEEE Transactions on Automatic Control, vol. 55 , no. 5 , pp. $1047-1058,2010.1$

[14] M. A. Lohe, "Non-Abelian Kuramoto models and synchronization," Journal of Physics A: Mathematical and Theoretical, vol. 42, no. 39, 395101, 2009. 1

[15] D. Nešić, A. R. Teel, and D. Carnevale, "Explicit Computation of the Sampling Period in Emulation of Controllers for Nonlinear SampledData Systems," IEEE Transactions on Automatic Control, vol. 54, no. 3, pp. 619-624, 2009. 1

[16] D. Elliott, Bilinear Control Systems - Matrices in Action. SpringerVerlag, 2009. 1

[17] E. D. Sontag, "An eigenvalue condition for sampled weak controllability of bilinear systems," Systems \& Control Letters, vol. 7, no. 4, pp. 313-315, 1986. 1

[18] I.-A. F. Ihle, M. Arcak, and T. I. Fossen, "Passivity-based designs for synchronized path-following," Automatica, vol. 43, no. 9, pp. 15081518, 2007. 1

[19] J. Giraldo, E. Mojica-Nava, and N. Quijano, "Synchronization of dynamical networks with a communication infrastructure: A smart grid application," in IEEE Conference on Decision and Control. Florence, Italy: IEEE, 2013, pp. 4638-4643. 1

[20] W. Sun, X. Yu, J. Lü, and S. Chen, "Synchronisation of directed coupled harmonic oscillators with sampled-data," IET Control Theory \& Applications, vol. 8, no. 11, pp. 937-947, 2014. 1

[21] P. J. McCarthy and C. Nielsen, "Local Synchronization of SampledData Systems on Lie Groups," 2017. 1, 4

[22] C. Lageman, J. Trumpf, and R. Mahony, "Gradient-like observers for invariant dynamics on a Lie group," IEEE Transactions on Automatic Control, vol. 55, no. 2, pp. $367-377,2010.2$

[23] M. Moakher, "Means and Averaging in the Group of Rotations," SIAM Journal on Matrix Analysis and Applications, vol. 24, no. 1, pp. 1-16, 2002. 2

[24] N. J. Higham, Functions of Matrices. Society for Industrial and Applied Mathematics, 2008. 3, 4

[25] S. Lang, Complex Analysis. New York, NY: Springer-Verlag, 1999, vol. 103. 3

[26] B. C. Hall, Lie Groups, Lie Algebras, and Representations. Springer International Publishing, 2015, vol. 222. 3

[27] C. Godsil and G. Royle, Algebraic Graph Theory. Springer-Verlag, 2001. 4

[28] R. Agaev and P. Chebotarev, "On the spectra of nonsymmetric Laplacian matrices," Linear Algebra and its Applications, vol. 399, no. 1-3, pp. 157-168, 2005. 4 